\title{
Continuous Passive Motion Machine for Elbow Rehabilitation
}

\author{
Hamzah Hussein Mohammed Al-Almoodi a,1 , Norsinnira Zainul Azlan a,2,*, Ifrah Shahdad a,3, \\ Nohaslinda Kamarudzaman ${ }^{\mathrm{b}, 4}$ \\ ${ }^{a}$ Department of Mechatronics Engineering, Kulliyyah of Engineering, International Islamic University Malaysia, Jalan \\ Gombak, 53100 Kuala Lumpur, Malaysia \\ ${ }^{\mathrm{b}}$ Sultan Ahmad Shah Medical Centre, Jalan Sultan Ahmad Shah, 25200 Kuantan, Pahang, Malaysia \\ ${ }^{1}$ hamza_h1h0@ hotmail.com; ${ }^{2}$ sinnira@iium.edu.my; ${ }^{3}$ ishahdad3@ gmail.com, ${ }^{4}$ norhaslinda@iium.edu.my \\ * Corresponding Author
}

\section{ARTICLE INFO}

\section{Article history}

Received 10 September 2021

Revised 21 October 2021

Accepted 23 October 2021

Keywords

Continuous Passive Motion $(\mathrm{CPM})$;

Upper Limb Rehabilitation

Machine;

Mechanism Design;

PID Controller;

Graphical User Interface (GUI)

\begin{abstract}
Continuous Passive Machines (CPM) facilitate patients in eliminating joint stiffness after surgery and lead to a faster and more efficient recovery. However, many previous CPM machined are mechanically complicated, expensive, and lack a user interface. This paper presents a new CPM machine for elbow flexion-extension and forearm pronation-supination. The machine is simple, low-cost, and equipped with Graphical User Interface (GUI). Its mechanism is designed so that it can be used on the left or right arms interchangeably. It is developed using aluminum, perspex, and steel rods. The electrical part of the machine consists of Arduino Uno to drive the motors and a potentiometer to measure the patients' Range of Motion (ROM). The GUI for setting the exercise parameters and monitoring the patients' progress has been developed using MATLAB software. The experimental results show that the machine has successfully provided the repetitive desired motions. The machine realizes elbow flexionextension and forearm pronation-supination movements with $0^{\circ}-135^{\circ}$ and $0^{\circ}-90^{\circ}$ ranges of motion (ROM), respectively. The machine is also capable of increasing the elbow joint's ROM by $5^{\circ}$ increments for the therapy. The results show that the machine has the potential to be used in hospitals and rehabilitation centers.
\end{abstract}

This is an open-access article under the CC-BY-SA license.

\section{Introduction}

Continuous passive motion (CPM) treatment is widely used in treating patients after various joint surgeries [1]-[8] to avoid joint stiffness and improve patient's recovery. It enhances the knee flexion range [9] and functionality [10] in the initial stage of postoperative for total knee arthroplasty (TKA). This type of treatment can also be combined with alternative medicines or therapy. In Korea, the treatment has been combined with Korean medicine for treating injuries at the knee, and this results in a more effective outcome [11]. In China, Cao et 
al. studies the effect of the treatment integrated with filiform fire-needling in the treatment for frozen shoulder [12].

Continuous Passive Motion (CPM) machine automates the CPM procedure, and it is important for helping the patients to regain their upper or lower limb functionality after a surgery or post joint trauma. It works by moving the patients' joints, which can be either the knee, elbow, shoulder, wrist, or fingers repetitively within a specified range of motion (ROM) without the patients having to move the limbs by themselves. Its continuous and passive movement avoids joint stiffness that is indicated by joint pain and limited movement. This joint stiffness may be caused by the adhesion of the joint capsule with the cartilage that is due to the reduction in the amount of synovial fluid, which acts as the joint's lubricant. This problem may occur due to the lack of joint movement following joint surgery or trauma [13]. CPM machines can also reduce pain, decrease the number of adhesions and amount of atrophy experienced by the supporting muscles [14], reduce scar tissue formation, and increase range of motion (ROM) and flexibility of the joints [15]. It also has the advantage of decreasing the duration of the patient's hospital stay, thereby reducing the therapists' workload and pressure.

Previous studies on CPM machines include the work in [13]-[29], Wang et al. developed a knee CPM machine. The machine is simple, and its motion is actuated by the DC geared motor and controlled through the Arduino microcontroller board [15]. Trochimczuk and Kuxmierowski [16] proposed a new CPM machine for the knee and performed its kinematic analysis. The machine is made of a four-bar linkage and a slider-crank mechanism. The mechanism operation was tested through computer simulation using Microsoft Visual Studio 2012 and Windows Presentation Foundation library [16]. Tangjitsitcharoen and Lohasiriwat [17] proposed a new form of CPM machine for total knee replacement therapy to solve structure redundancy and mechanism complexity in the existing CPM machine. The suitability of the machine's measurement was verified by finite element analysis [17].

A Continuous Passive Motion (CPM) focusing on the fingers and wrist joints was developed by Almusawi and Geza [19] and Almusawi et al. [20]. The proposed machine was cost-efficient and can be used by both right and left arms. Its structure is based on the grounded-exoskeleton concept, and it is able to realize the flexion and extension movements of the fingers and radial and ulnar deviation of the wrist. The mechanism was built using a lead screw and a dual-sided actuator [19]. Fu et al. [21] developed a CPM machine that is portable. It can be used with various finger sizes. The machine can attain the desired fingers' range of motion, amount of force to be exerted, and angular speed accurately [8]. In the CPM machine for the wrist joint developed by Noviyanto et al. [13], the angular displacement and speed can be adjusted according to the therapy requirement. The machine is attached to a chair and able to help the patients to conduct continuous repetitive shoulder flexion and horizontal abduction motions [13]. Park et al. [24] extended the CPM to be used for mirror therapy for arm rehabilitation. CPM machines can also be controlled by the motor imagery-based brain-computer interface (BCI) [23] and actuated by pneumatic muscles [25].

Numerous lower limb CPM machines can be found in Malaysian hospitals. However, a relatively smaller number of CPM machines for the upper limb are used in these hospitals. This situation, among others, is contributed by the lower number of commercial CPM machines for the upper limb, bulky design of many upper limb CPM machines with no anatomical adjustability to cater for different sizes of individuals, and also the lack of a user-friendly interface to make the therapy to be more experiential and immersive for the patients.

The contribution of this paper is a new design of 2 degrees of freedom (DOF) CPM machine for elbow flexion-extension and forearm pronation-supination that is simple, lightweight, low cost. The mechanism design enables the machine to be used interchangeably by the left and right arms. A new Graphical User Interface (GUI) has been specifically developed for the machine to allow the therapists and patients to observe and monitor the treatment progress. The CPM machine is programmed to provide the option for the therapist to set or increase the 
desired Range of Motion (ROM) of the joints during the rehabilitation exercise gradually in small increments as in the manual rehabilitation. A prototype of the machine has been developed using aluminum, perspex, and steel rods. A potentiometer is utilized to measure patients' joints ROM. The required joint angular displacement and speed during the exercise can be set by the therapists a priori.

This paper is organized as follows. Section 2 presents the mechanism design of the CPM machine for elbow rehabilitation. The mathematical modeling and PID controller for controlling the speed of elbow joint movement are described in Section 3 and Section 4, respectively. The explanation of the developed GUI is provided in Section 5. Section 6 discusses the results obtained, and finally, the conclusion is drawn in Section 7.

\section{Mechanism Design}

This section describes the mechanical design and electrical connection of the CPM machine.

\subsection{Mechanical Design}

The proposed CPM machine has been developed with two degrees of freedom (D.O.F.) for elbow flexion-extension motion and forearm pronation -supination movement. Fig. 1 shows the main components of the CPM machine. The CPM machine consists of a table that is made of aluminum, where the affected human arm is fastened to the machine using a Velcro strap. The table movement is actuated by a DC motor which is placed below the table to perform the flexion-extension motion. The table is fixed to an aluminum L-shaped linkage. This linkage is connected to the holder, round disk (Round 1), and four pieces of lead screws. An arc (Round 2) and a handle are connected to the leadscrew, and a servo motor is mounted at the back of the disk (Round 1). These rounds have been made using a 3D printer. During the therapy, the patients hold the handle, and the servo motor moves the holder, Round 1, leadscrew, Round 2, and handle, together with the patient's forearm for pronation and supination movement. The table is mounted on two layers of aluminum supports at the top and bottom and one layer of perspex in the middle. The middle layer is designed with a slot to enable the L-shape linkage to rotate, and it is lubricated with grease to reduce the friction between the linkage and the table. A potentiometer is connected to the DC motor below the table using a 1:1 gear to measure the angular displacement and range of motion (ROM) of the elbow joint for the flexion-extension movement. The overall cost of the machine is RM 480.00. Fig. 2 and Fig. 3 illustrate the developed CPM machine prototype for the elbow and the human arm position while using the machine for therapy, respectively.

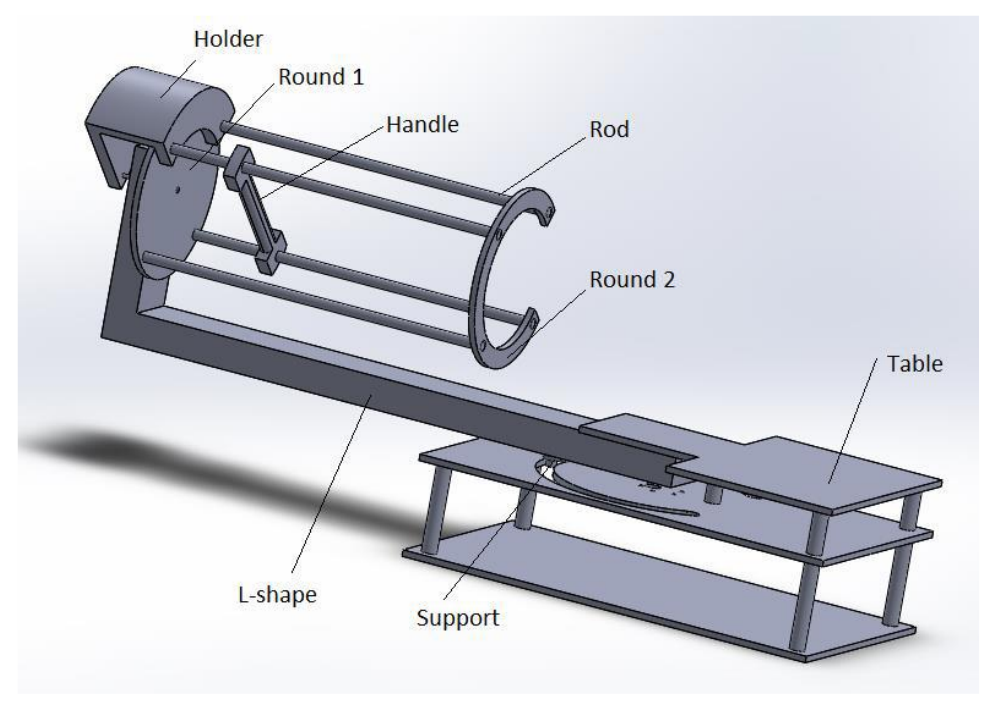

Fig. 1. Main components of the CPM machine 


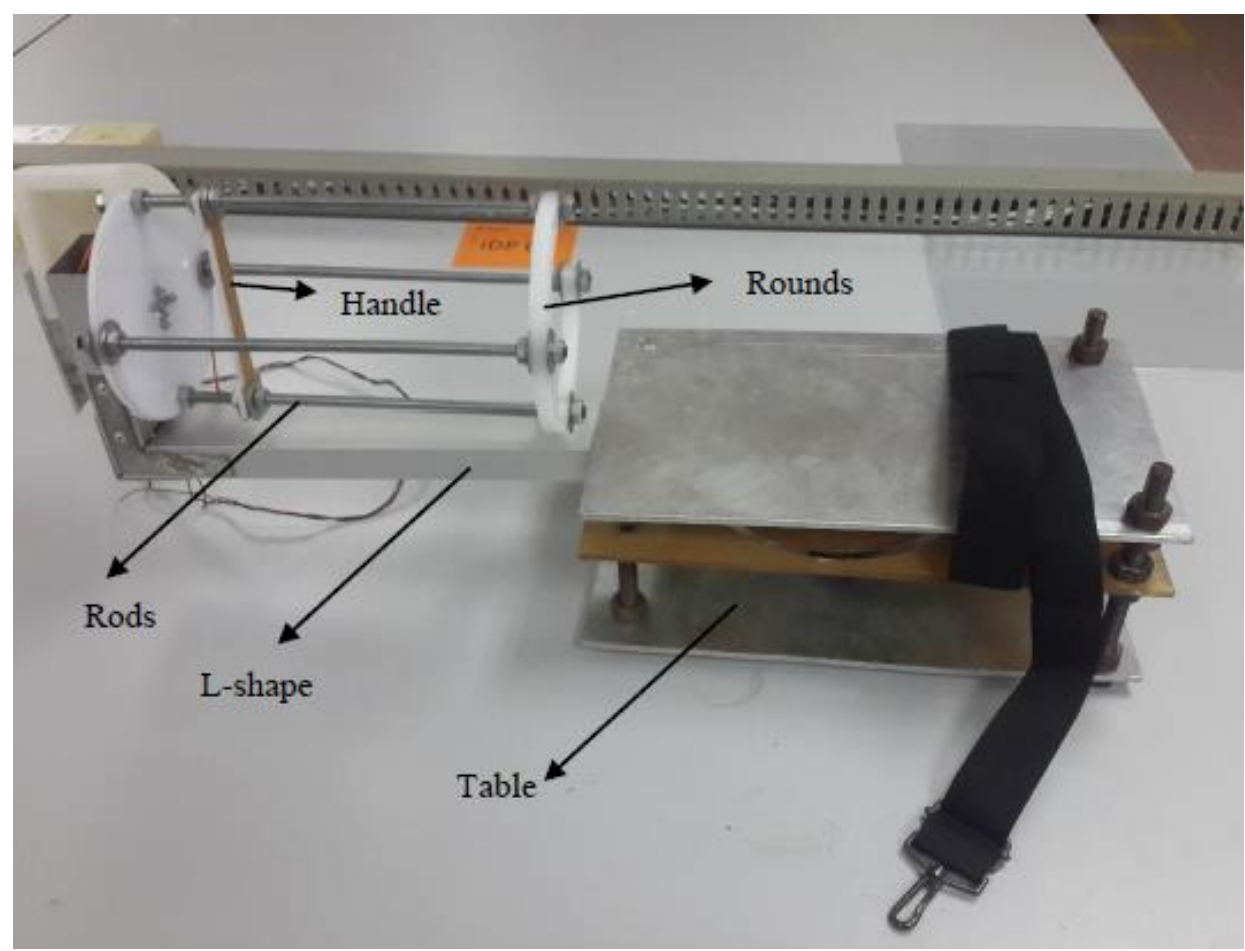

Fig. 2. Proposed CPM machine prototype

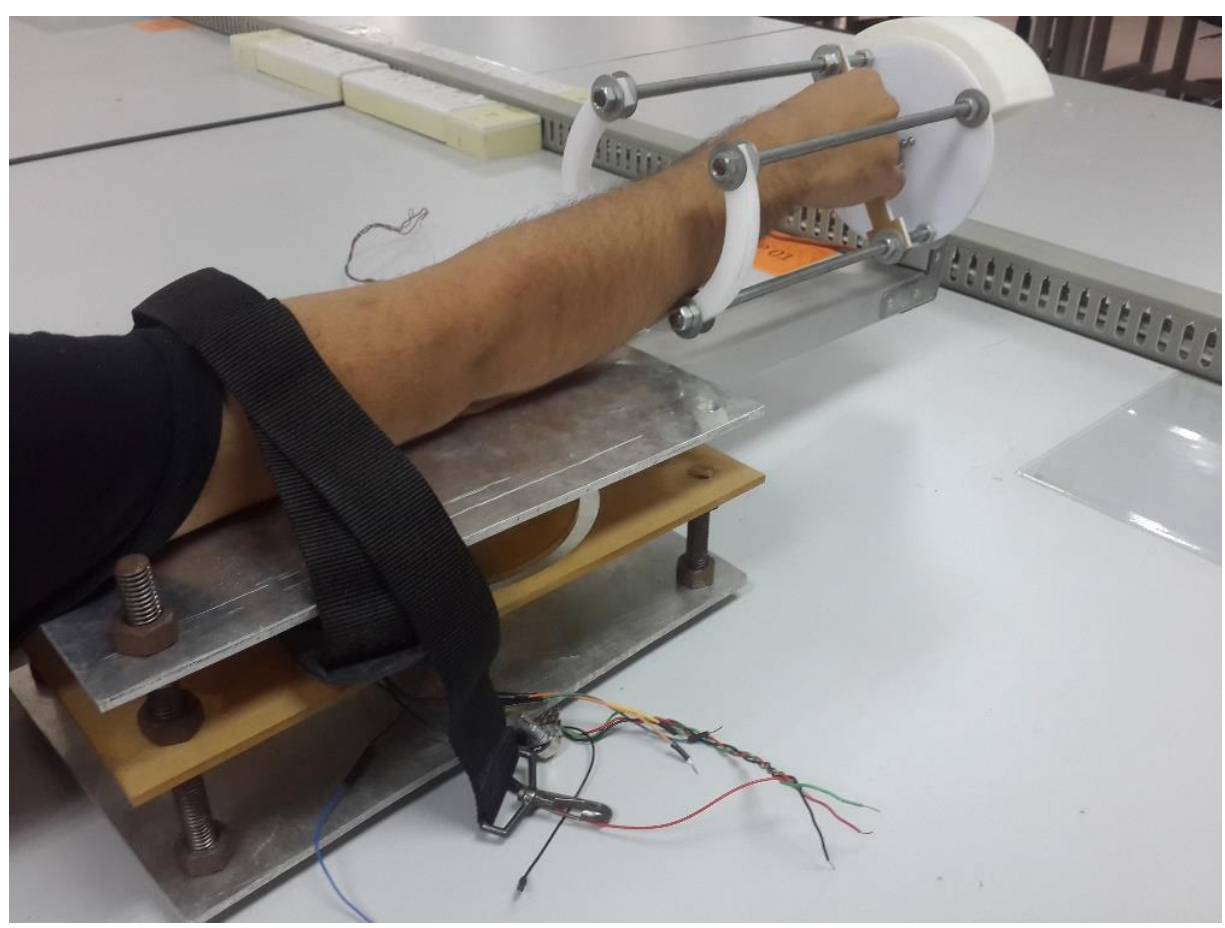

Fig. 3. Human arm while using the CPM machine at full extension

\subsection{Electrical Connection}

Fig. 4 shows the electrical connection of the system, consisting of an Arduino Uno as the microcontroller board, a DC Motor, a servomotor, a potentiometer, a push button, a motor driver (L293D), and a power supply. The electrical part of the system provides the necessary input for the machine movement, measures the angular displacements during both flexion-extension and pronation-supination motions, and also ensures the patients' safety while using the machine. The motor driver, L293D, is connected to the power supply, 3 of its pins are connected directly 
to the digital inputs of the Arduino, and its other two pins are fixed to the DC motor. During the flexion-extension therapy, the patient will press the push button if she/he feels any pain, and the motor will stop from rotating and then turns in the opposite direction. By this, the machine can avoid any injuries and learn the patients' range of motion (ROM).

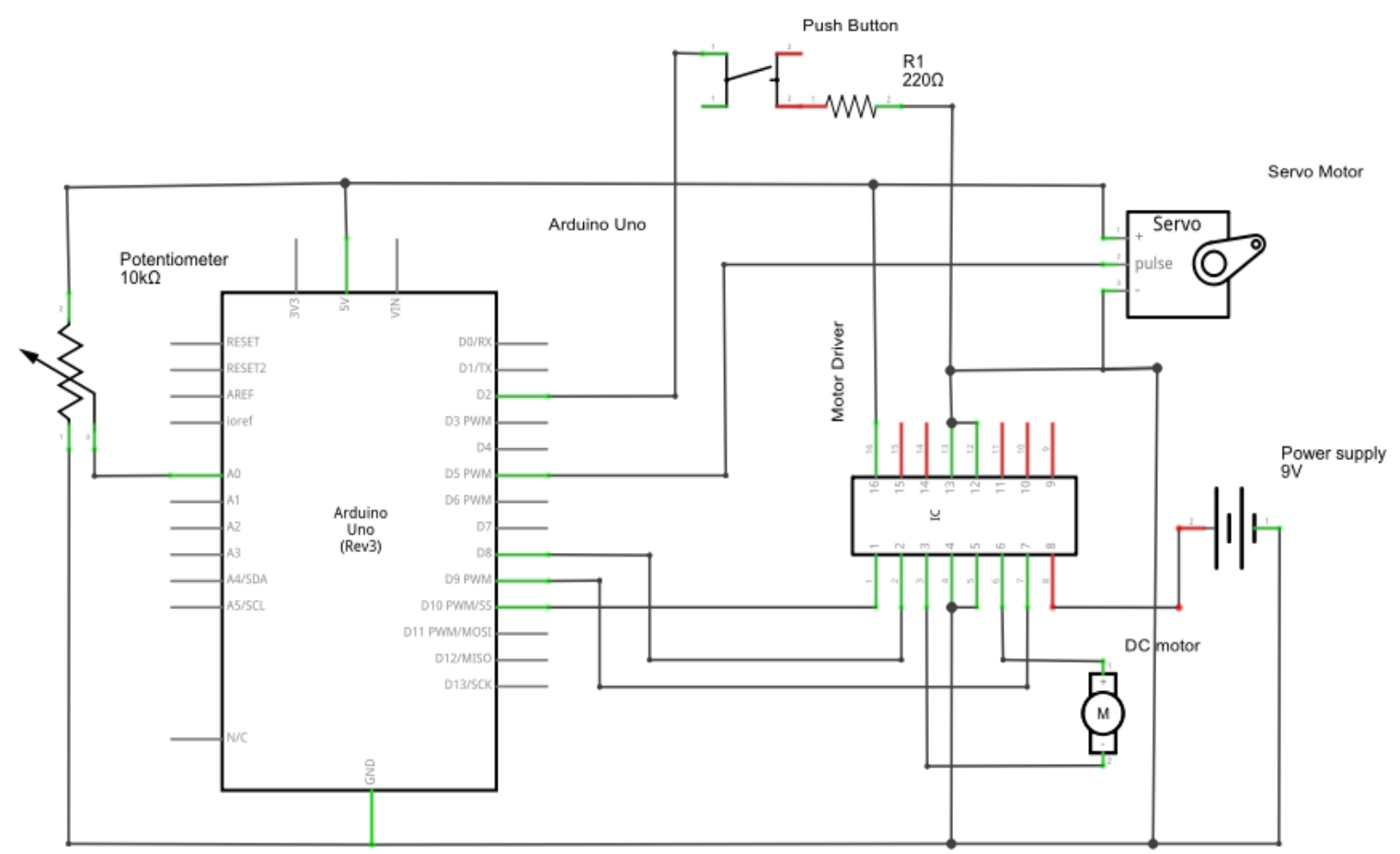

Fig. 4. The electrical connection of the CPM Machine

\section{Mathematical Model}

\subsection{Mathematical Model of the Mechanical Linkages}

The mathematical model is developed for controlling the machine speed during therapy. In the model, the whole structure that is attached to the L-shape linkage ad itself is lumped as a single link for simplicity. The mathematical equation for modeling a mechanical system can be written as

$$
T(t)=\frac{\partial}{\partial t}\left(\frac{\partial L}{\partial \omega}\right)-\frac{\partial L}{\partial \theta}
$$

Where $T$ is torque of the link, $L$ is Lagrange equation, $\theta$ is angular displacement of the link, $\omega$ is angular velocity of the link and $t$ is time.

The Lagrange equation can be expressed by

$$
L=K-P,
$$

where $K$ is the kinetic energy and $P$ is the potential energy. $K$ is equal to

$$
K=\frac{1}{2} I \omega^{2}(t)
$$

and $I$ is moment of inertia and simplified as

$$
I=\frac{1}{3} m l^{2}
$$


The potential energy is zero since the motor is rotating horizontally. Substituting (3) and (4) into (2), the Lagrange equation becomes

$$
L=\frac{1}{2}\left(\frac{1}{3} m l^{2}\right) \omega^{2}(t)
$$

Differentiating (5) to get the partial derivative of $L$ with respect to $\omega$ yields

$$
\frac{\partial L}{\partial \omega}=\frac{1}{3} m l^{2} \omega(t)
$$

Differentiating (6) with respect to $t$ gives,

$$
\frac{\partial}{\partial t}\left(\frac{\partial L}{\partial \omega}\right)=\frac{1}{3} m l^{2} \alpha(t)
$$

where $\alpha$ is the angular acceleration. Substituting (7) into (1) results in

$$
T(t)=\frac{1}{3} m l^{2} \alpha(t)
$$

\subsection{Mathematical Model of the Electrical Motor}

Using Kirchhoff Voltage Law (KVL), the equation for the voltage in a motor can be written as

$$
V(t)=R i(t)+L_{\text {ind }} \frac{d i}{d t}+k_{b} \omega(t)
$$

where $i(t)$ is the current, $R$ is the resistance, $L_{\text {ind }}$ is the inductance, and $k_{b}$ is the back emf constant. The dynamic equation of the mechanical part of the motor can be written as

$$
J \alpha(t)+b \omega(t)+T(t)=k_{t} i(t)
$$

where $J$ is the moment of inertia, $b$ is the friction factor, and $k_{t}$ is the motor torque constant. Substituting (8) into (10) gives

$$
J \alpha(t)+b \omega(t)+\frac{1}{3} m l^{2} \alpha(t)=k_{t} i(t)
$$

\section{PID Controller}

The equation for PID controller to control for the elbow flexion-extension movement speed in the CPM machine is given as

$$
u(t)=K_{p} e(t)+K_{d} \frac{d e}{d t}+K_{i} \int_{0}^{t} i(\tau) d \tau
$$

where $u(t)$ is the input voltage to the motor $e(t)$ is the difference between the desired and actual speed of the link. The $K_{p}, K_{d}$ and $K_{i}$ are the proportional, derivative and integral gains, respectively [30].

\section{Graphical User Interface (GUI) Development}

The developed graphical user interface (GUI) enables the user/therapist to enter the exercise setting and monitor the patient's movement during the therapy. It has been constructed using MATLAB software together with Arduino IDE. The user interface for the CPM machine starts with a dialog box prompting the user to start the therapy, as shown in Fig. 5. 
After pressing the "START" in Fig. 5, window 1, as shown in Fig. 6, will appear. The window will display four buttons which are "Measure," "Exercise," "Settings," and "Exit." Pressing the "Settings" button will lead to Window 2, as illustrated in Fig. 7, which will allow the therapist to enter the therapy setting. Clicking the "Measure" button will prompt Window 3 , as shown in Fig. 8, to measure the patients of a range of motion (ROM). Window 4, as depicted in Fig. 9, will appear once the "Exercise" button is pressed to start the therapy, and finally, the "Exit" button is used to stop the whole therapy session.

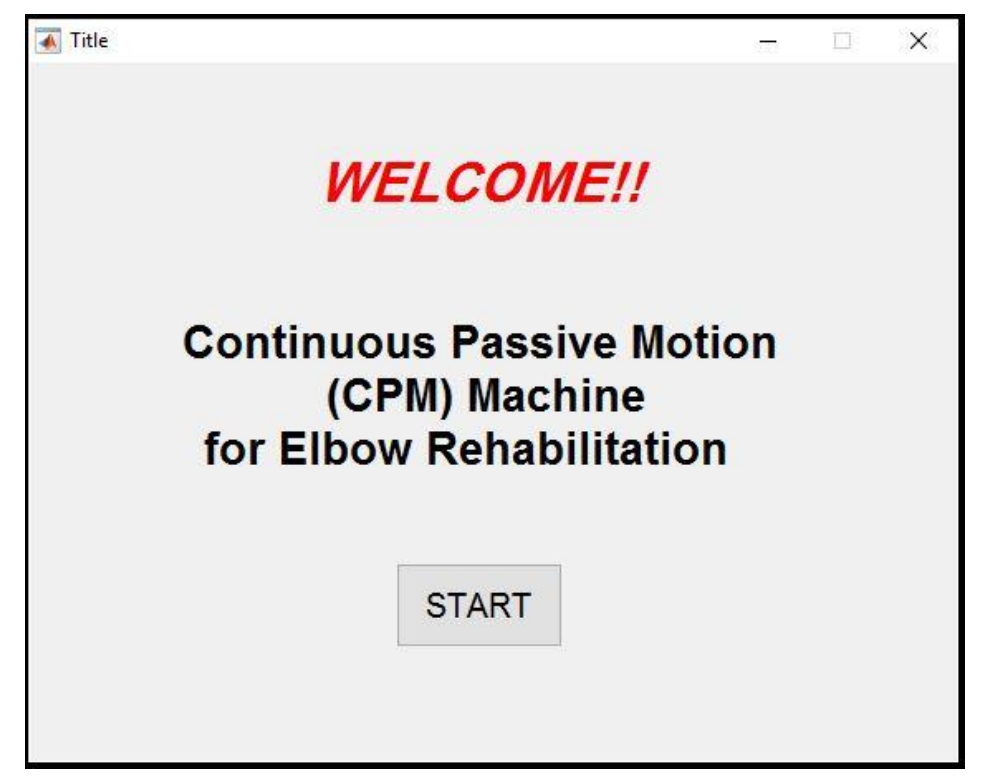

Fig. 5. Welcome window

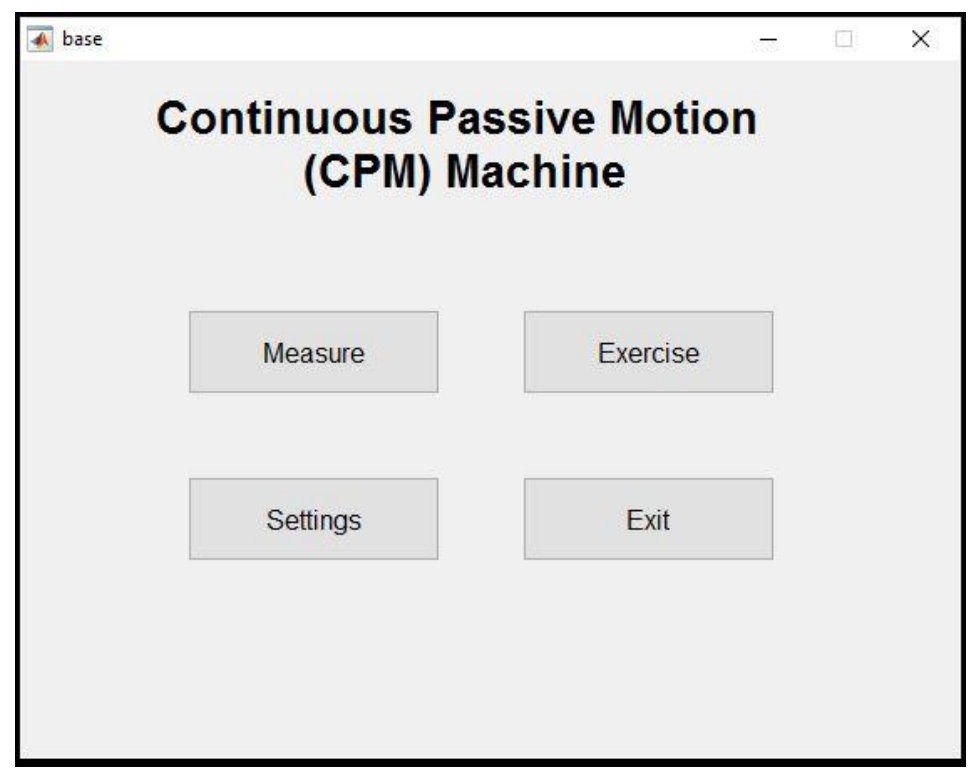

Fig. 6. CPM Window 1

In Window 2, the therapist can enter whether the therapy will be done on the right or left arm, which movement will be realized (flexion-extension or pronation-supination), the speed of the motor movement, and also the duration of the exercise.

Upon the selection of the "Measure" option (Window 3), as shown in Fig. 8, the machine will measure the patient's range of motion (ROM) or the maximum angle that he/ she can move his/ her arm for the type of movement that had been chosen in the Setting window a priori. The therapist needs to press the "START" button, and the patients need to move his/ her elbow 
or forearm to begin the measurement process. Once the patient has reached his or her maximum limit, the therapist needs to press the "STOP" button. The result will be displayed graphically. After the measurement of the ROM, the therapist can start the exercise by pressing the "Exercise" button or return to the previous window by pressing the "Back" button.

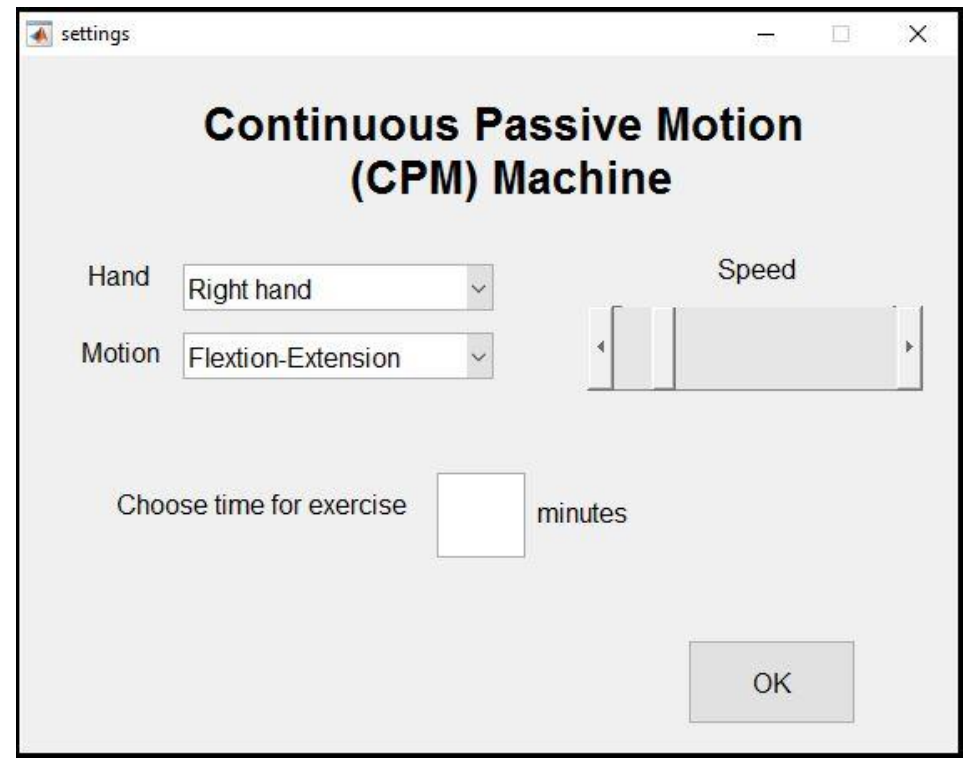

Fig. 7. CPM Window 2 for "Setting" option

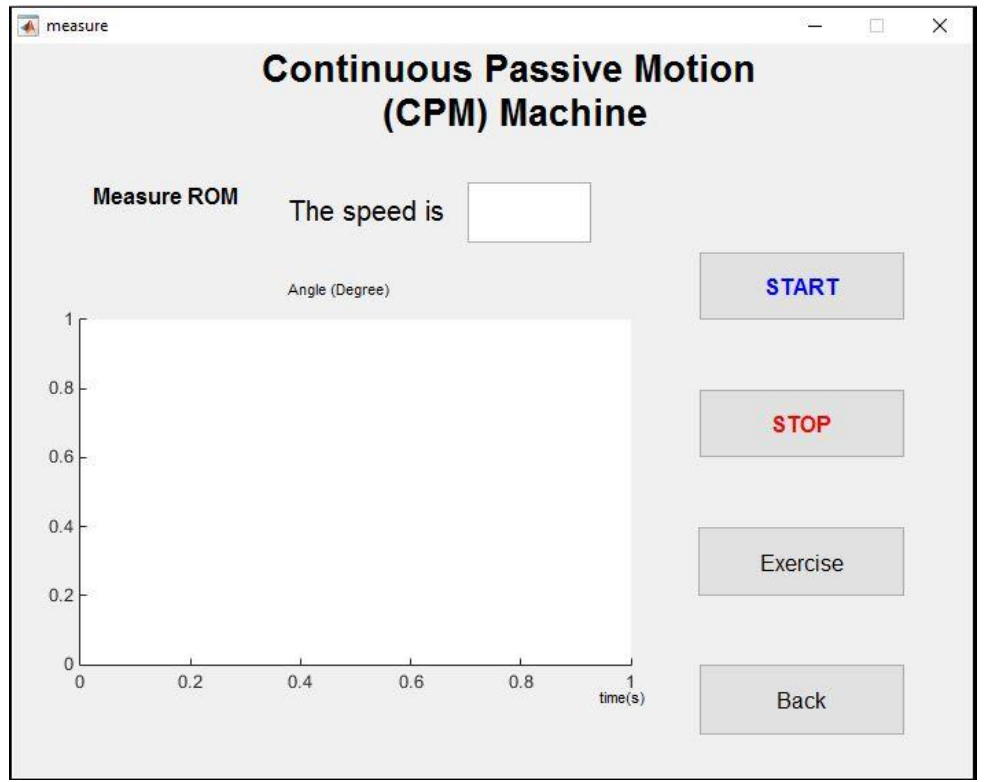

Fig. 8. CPM Window 3 for "Measure" option

In the "Exercise" window, the therapist can press the "START" button to begin the exercise session based on the type of motion, speed, and duration of the exercise that have been keyed in the "Settings" window. The exercise will be continued until the duration ends. The therapist may also terminate the exercise at any time by pressing the "STOP" button and continue the exercise by clicking the "Continue" button. The therapist may click the "Add 5 Degree" option to increase the patient's ROM in a small increment in the therapy. The patients' elbow or forearm movement graph during the therapy is displayed in this window. Pressing the "Back" button leads the therapist to the previous window. 


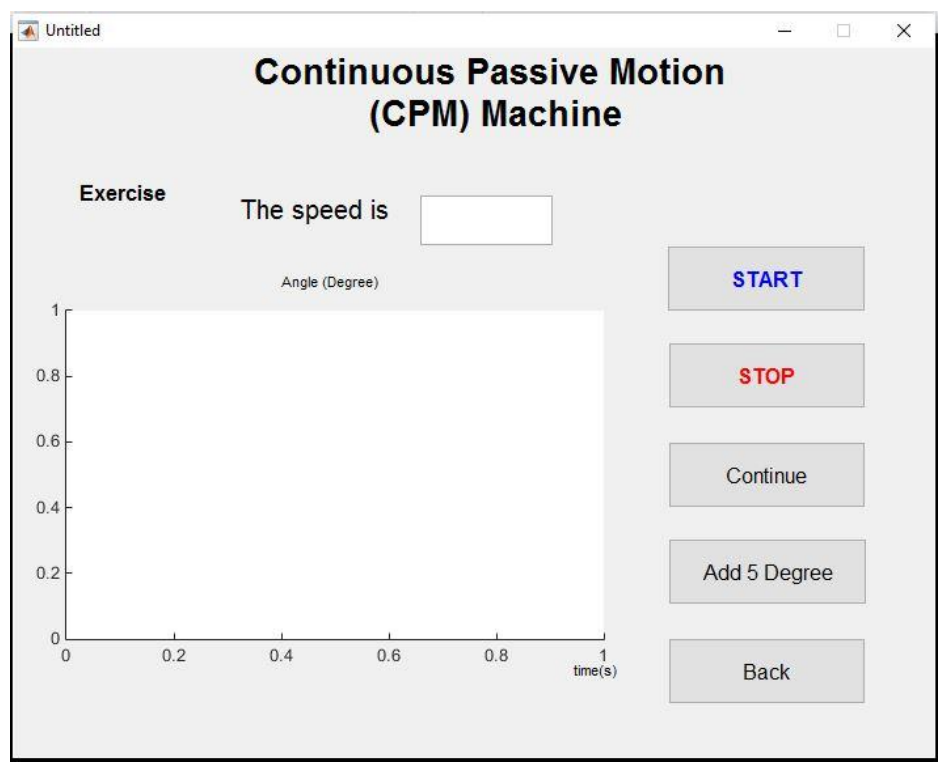

Fig. 9. CPM Window 4 for the "Exercise" option

\section{Results and Discussion}

\subsection{PID Control Simulation Results}

The simulation test focuses on the speed control of the CPM machine's flexion-extension motion described in Sections 3 and 4. In this test, the desired speed is set to be $72.5^{\circ} / \mathrm{s}$ which is equivalent to $1.265 \mathrm{rad} / \mathrm{s}$ since it is required for the machine to move the full range of movement of $145^{\circ}$ within 2 seconds. It is also desired for the machine to achieve zero percentage overshoot and steady-state error less than 0.005 rad.

The PID parameters, $K_{p}, K_{d}$ and $K_{i}$ have tuned manually to 66,3 , and 5, respectively. Increasing the value of $K_{p}$ makes the actual output closer to the desired position, whereas in raising the values of $K_{i}$ and $K_{d}$ reduces the steady-state error and overshoot percentage, respectively. The system response under the PID is shown in Fig. 10. From the figure, it can be seen that the system reaches the desired speed with settling time less than $0.2 \mathrm{~s}$ and percentage overshoot less than 1\% under the PID controller.

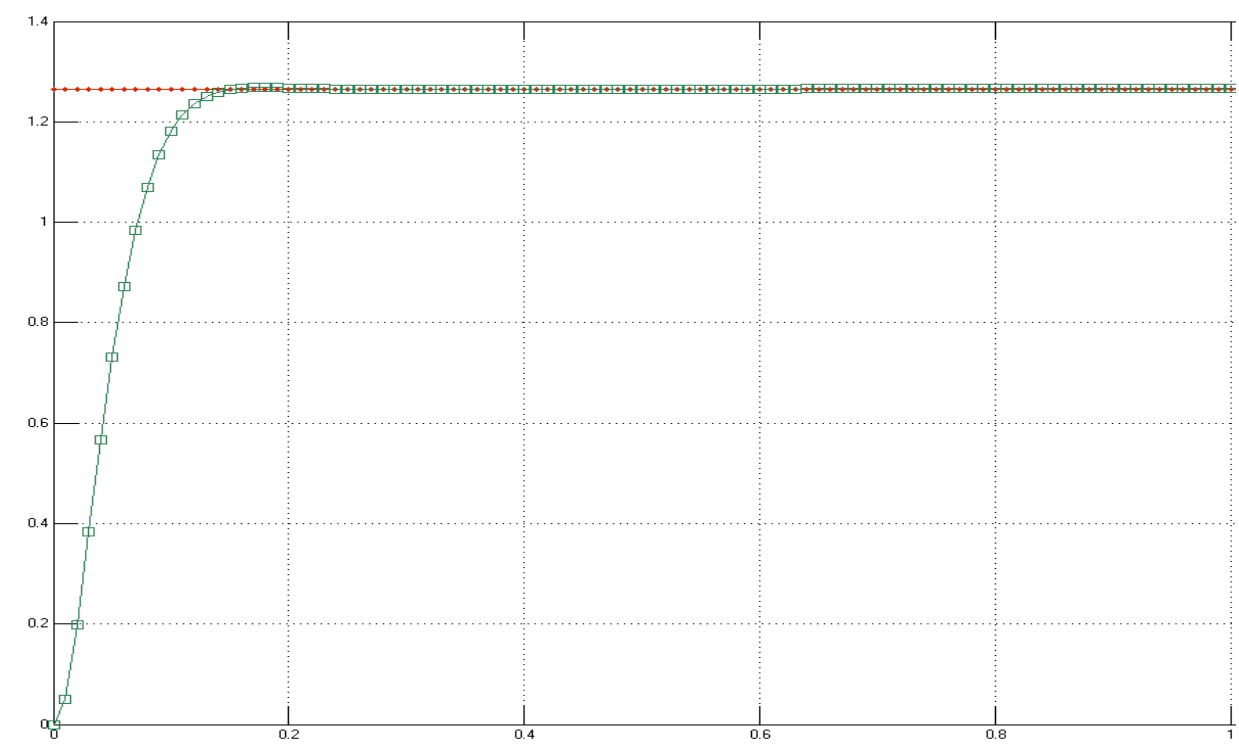

Fig. 10. System response under PID controller 


\subsection{CPM Machine Experimental Test Results}

The prototype of the CPM machine has been developed and tested for ROM measurement and flexion-extension and pronation-supination exercises. The results from the CPM machine for measuring the patient's ROM for flexion-extension motion are depicted in Fig. 11. The figure indicates that the machine has measured a ROM of $-82^{\circ}$ for this particular subject. From the figure, it can be seen that the proposed machine has successfully taken and displayed the ROM measurement in real-time for the therapy.

For the flexion-extension motion, the resistance of the potentiometer varies with the motor movement, and the value is transferred to the microcontroller in terms of voltage change. The Arduino microcontroller board converts the voltage change into digital output ranging from 0 1023 as it has a 10-bit Analog to Digital Converters (ADC). Thus, if the resistance of the potentiometer is set to the middle, the Arduino will show a value of 512. If the right hand is under therapy, the angular movement will be from $0^{\circ}$ to $135^{\circ}$ for the full flexion, and then the linkage will reverse its movement. If the left hand is under training, the angular position will be from $0^{\circ}$ to $-135^{\circ}$.

Fig. 12 shows the extension-flexion exercise that was performed with a speed of 28.5 degree/s. The DC motor rotates downwards from $0^{\circ}$ to $-82^{\circ}$ repetitively for 2 minutes based on the ROM measured in Fig. 11. If the therapist would like to increase the ROM in the exercise with an increment of $-5^{\circ}$, then the CPM machine will rotate up to $-87^{\circ}$.

The forearm pronation-supination movement has been carried out using the servo motor, which rotates between $0^{\circ}$ to $90^{\circ}$. Fig. 13 shows the GUI display capturing the subject's forearm pronation-supination motion during the exercise with the ROM of $67^{\circ}$ to $90^{\circ}$ at the speed of $-3.2^{\circ} / \mathrm{s}$.

The experimental results show that the proposed CPM machine has successfully measured the patient's ROM and performed the repetitive elbow flexion-extension and forearm pronation-supination motions as required. The continuous passive treatment can be done on the left or right arm interchangeably using the same machine. It can also be seen from the results that the GUI functions properly in providing the interface for the therapists to key in the input for the treatment settings. It also provides a clear description of the patients' treatment progress and achievement for easy monitoring and recording.

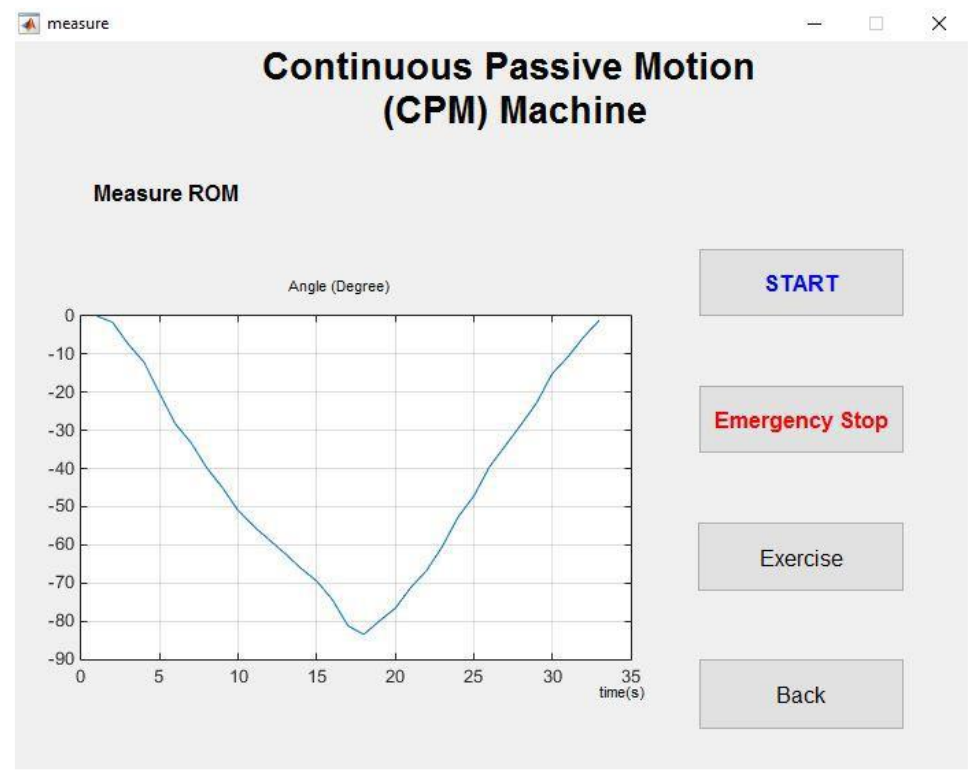

Fig. 11. ROM measurement displayed on the GUI 


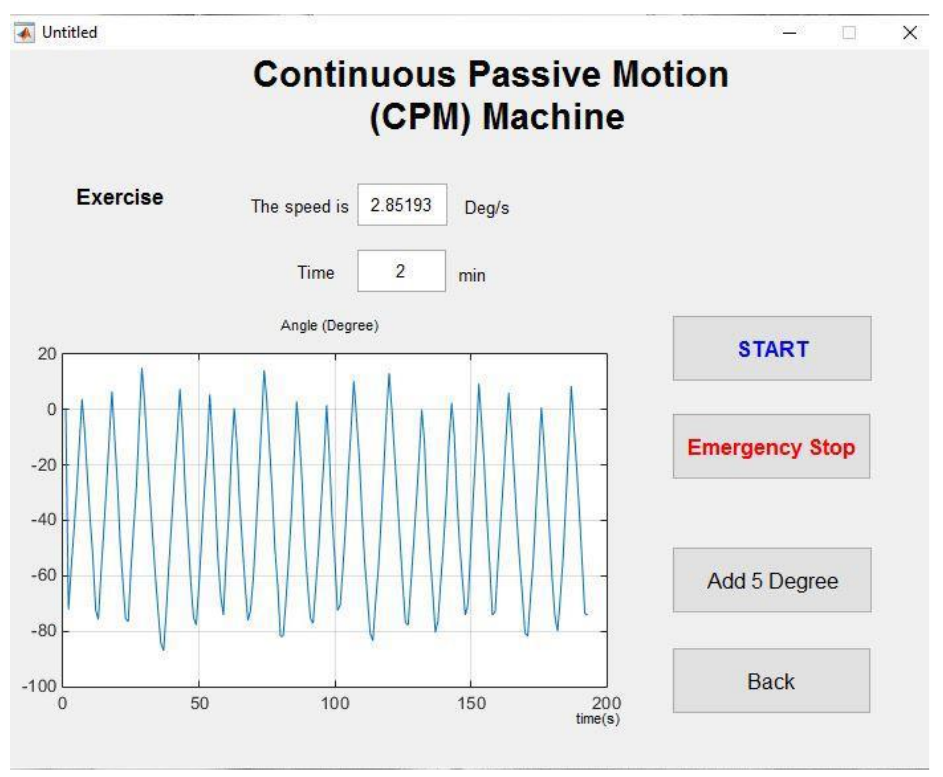

Fig. 12. Elbow flexion-extension motion during the exercise displayed by the GUI.

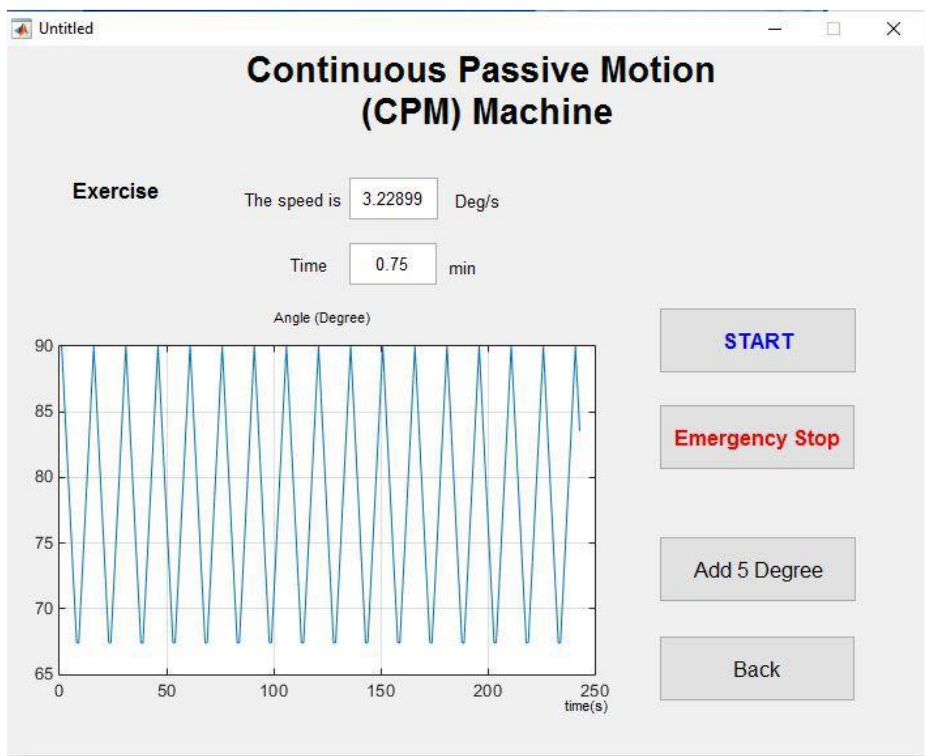

Fig. 13. Forearm pronation-supination motion during the exercise displayed by the GUI.

\section{Conclusion}

In this study, a 2 DOF CPM machine has been developed and tested. The machine is simple, low-cost, and equipped with GUI. Left or right arm rehabilitation treatment is possible using the same CPM machine due to the proposed mechanism design. The experimental results show that the machine is capable of facilitating the patients' rehabilitation therapy to regain their elbow flexion-extension and forearm pronation-supination functions as desired. The ROM for the elbow and forearm movements are $0^{\circ}-135^{\circ}$ and $0^{\circ}-90^{\circ}$ respectively, and the span of the joint displacement during the exercise can be increased or decreased by $5^{\circ}$. The PID controller has successfully controlled the speed of the DC motor in realizing the elbow flexion-extension movement. The GUI has been developed using MATLAB, and it allows the therapist to set the exercise parameters and monitor the patients' progress. Future works involve: (1) improving the machine's mechanism to accommodate patients among children, (2) extending the machine 
for fingers rehabilitation, (3) enhancing the GUI with games to encourage and increase the patients' motivation, and (4) clinical studies with real patients.

Funding: This research was funded by INTERNATIONAL ISLAMIC UNIVERSITY MALAYSIA, grant number P-RIGS18-019-0019.

\section{References}

[1] B. Stolz, C. Grim, C. Lutter, K. Gelse, M. Schell, B. Swoboda, H. D. Carl, and T. Hotfiel "Assessing Foot Loads in Continuous Passive Motion (CPM) and Active Knee Joint Motion Devices," Sportverletz Sportschaden, vol. 35, no. 1, pp. 18-23, 2021. https://doi.org/10.1055/a-0648-8699

[2] T. D'Amore, S. Rao, J. Corvi, R. A. Jack, F. P. Tjoumakaris, M. G. Ciccotti, and K. B. Freedman, "The Utility of Continuous Passive Motion After Anterior Cruciate Ligament Reconstruction: A Systematic Review of Comparative Studies," Orthopaedic Journal of Sports Medicine, vol. 9, no. 6, pp. 1-9, June 2021. https://doi.org/10.1177/23259671211013841

[3] S. S. Kantar and I. Fusaro, "Rehabilitation, Use of Elbow Braces, and Continuous Passive Motion After Elbow Arthroplasty," In Castoldi F., Giannicola G., Rotini R. (eds) Elbow Arthroplasty. Springer, Cham., pp. 0303-320, 2020. https://doi.org/10.1007/978-3-030-14455-5_29

[4] J. T. Bram, A. J. Gambone, C. J. DeFrancesco, B. M. Striano, and T. J. Ganley, "Use of Continuous Passive Motion Reduces Rates of Arthrofibrosis After Anterior Cruciate Ligament Reconstruction in a Pediatric Population," Orthopaedics, vol. 42, no. 1, pp. 81-85, November 2018. https://doi.org/10.3928/01477447-20181120-04

[5] J. Viveen, J. N. Doornberg, I. F. Kodde, P. Goossens, K. L. M. Koenraadt, B. The, and D. Eygendaal, "Continuous Passive Motion and Physical Therapy (CPM) versus Physical Therapy (PT) versus Delayed Physical Therapy (DPT) After Surgical Release for Elbow Contractures; A Study Protocol for a Prospective Randomized Controlled Trial," BMC Musculoskeletal Disorder, vol. 18, no. 1, pp. 1-7, November 2017. https://doi.org/10.1186/s12891-017-1854-0

[6] M. Schulz, K. Bernhard, W. Röder, and K. Sander, "Randomized, Prospective, Monocentric Study to Compare the Outcome of Continuous Passive Motion and Controlled Active Motion After Total Knee Arthroplasty," Technology and Health Care, vol. 26, no. 3, pp. 499-506, June 2018. https://doi.org/10.3233/THC-170850

[7] M. C. Chen, C. C. Lin, J. Y. Ko, and F. C. Kuo, "The Effects of Immediate Programmed Cryotherapy and Continuous Passive Motion in Patients After Computer-assisted Total Knee Arthroplasty: A Prospective, Randomized Controlled Trial," Journal of Orthopaedic Surgery and Research, vol. 15, article no. 379, 2020. https://doi.org/10.1186/s13018-020-01924-y

[8] C. D. Liao, Y. C. Huang, Y. S. Chiu, and T. H. Liou, "Effect of Body Mass Index on Knee Function Outcomes Following Continuous Passive Motion in Patients with Osteoarthritis After Total Knee Replacement: A Retrospective Study," Physiotherapy, vol. 103, no. 3, pp. 266-275, September 2017. https://doi.org/10.1016/j.physio.2016.04.003

[9] N. Wirries, M. Ezechieli, K. Stimpel, and M. Skutek, "Impact of Continuous Passive Motion on Rehabilitation Following Total Knee Arthroplasty," Physiotherapy Research Internationals, vol. 25, no. 4, e1869, September 2020. https://doi.org/10.1002/pri.1869

[10] P. Z. Hasubhai, D. B. Dibyendenurayan, and A. T. Ramalingam, "Effectiveness of Conventional Physiotherapy along with Continuous Passive Motion after Total Knee Arthroplasty", Indian Journal of Physiotherapy and Occupational Therapy, vol. 11, no. 4, pp. 195-200, 2017. https://doi.org/10.5958/0973-5674.2017.00145.9

[11] H. J. Ha and M. S. Oh, "A Systemic Review of Korean Medical Treatment Combined with Continuous Passive Motion Therapy on the Knee Joint Postoperative Rehabilitation," Journal of Korean Medicine Rehabilitation, vol. 27, no. 3, pp. 33-45, July 2017. https://doi.org/10.18325/jkmr.2017.27.3.33 
[12] Y. Cao, C. Chen, W. J. Zhou, F. Zhong, and W. Zhang, "Clinical Observation on Filiform Fire-Needling Plus Continuous Passive Motion Therapy for Frozen Shoulder," Journal of Acupuncture and Tuina Science, vol. 18, pp. 281-285, August 2020. https://doi.org/10.1007/s11726-020-1189-5

[13] A. H. Noviyanto, L. D. Septilianingtyas, and Rahmawati, D. "Design of a Continuous Passive Motion (CPM) Machine for Wrist Joint Therapy," Journal of Robotics and Control (JRC), vol. 2, no. 4, pp 311-315, 2021. https://doi.org/10.18196/jrc.2498

[14] J. Rattarojpan and S. Umchid, "Design and development of touch screen based Continuous Passive Motion device for knee rehabilitation, $4^{\text {th }} 2011$ Biomedical Engineering International Conference, 2012, pp. 237-241. https://doi.org/10.1109/BMEiCon.2012.6172060

[15] W. K. Wang, M. G. Nabila, Z. Mohamad, and W. R. W. Omar, "The Development of Spectra Knee CPM Moving Train Walk Review," American Journal of Science, Engineering and Technology, vol. 2, no. 2, pp. 57-61, March 2017. http://www.ajoset.com/article/325/10.11648.j.ajset.20170202.11

[16] R. Trochimczuk and T. Kuxmierowski, "Kinematic Analysis of CPM Machine Supporting to Rehabilitation Process after Surgical Knee Arthroscopy and Arthroplasty," International Journal of Applied Mechanics and Engineering, vol. 19, no. 4, pp. 841-848, 2014. https://doi.org/10.2478/ijame-2014-0059

[17] S. Tangjitsitcharoen and H. Lohasiriwat, "Redesign of a Continuous Passive Motion Machine for Total Knee Replacement Therapy,” Journal of Health Research, vol. 33, no. 2, pp. 106-118, 2019. https: //doi.org/10.1108/JHR-06-2018-0024

[18] A. H. Noviyanto, "Saving the Moving Position on the Continuous Passive Motion Machine for Rehabilitation of Shoulder Joints," International Journal of Applied Sciences and Smart Technologies, vol. 1, no. 2, pp. 121-128, 2019. https://doi.org/10.24071/ijasst.v1i2.1921

[19] H. Almusawi and H. Géza, "Design and Development of Continuous Passive Motion (CPM) for Fingers and Wrist Grounded-Exoskeleton Rehabilitation System," Applied Sciences, vol. 11, no. 2, pp. 1-23, January 2021. https://doi.org/10.3390/app11020815

[20] H. Almusawi, S. A. Afghan, and H. Géza, "Designing the Mechanical Parts of a Low-Cost Hand Rehabilitation CPM Device for Stroke Patients," Lecture Notes in Electrical Engineering, vol. 505, pp. 60-66, 2018. https://doi.org/10.1007/978-3-319-91334-6_9

[21] Y. Fu, P. Wang, S. Wang, H. Liu, and F. Zhang, "Design and development of a portable exoskeleton based CPM machine for rehabilitation of hand injuries," 2007 IEEE International Conference on Robotics and Biomimetics (ROBIO), 2007, pp. 1476-1481. https://doi.org/10.1109/ROBIO.2007.4522382

[22] J. Li, K. Wang and F. Li, "Reduction of Torque Ripple in Consequent-Pole Permanent Magnet Machines Using Staggered Rotor," in IEEE Transactions on Energy Conversion, vol. 34, no. 2, pp. 643-651, June 2019. https://doi.org/10.1109/TEC.2018.2873318

[23] R. R. Lu, M. X. Zheng, J. Li, T. H. Gao, X. Y. Hua, S. H. Huang, J. G. Xu, and Y. Wu, "Motor Imagery based Brain-computer Interface Control of Continuous Passive Motion for Wrist Extension Recovery in Chronic Stroke Patients," Neuroscience Letters, vol. 718, p. 134727, January 2020. https://doi.org/10.1016/j.neulet.2019.134727

[24] T. Park, M. Lee, T. Jeong, Y. Shin, and S. M. Park, "Quantitative Analysis of EEG Power Spectrum and EMG Median Power Frequency Changes after Continuous Passive Motion Mirror Therapy System," Sensors, vol. 20, no. 8, pp 1-16, April 2020. https://doi.org/10.3390/s20082354

[25] A. Deaconescu and T. Deaconescu, "Pneumatic Equipment for Ankle Rehabilitation by Continuous Passive Motion," Mechanisms and Machine Science, vol. 84, pp. 13-21, June 2020. https://doi.org/10.1007/978-3-030-48989-2_2

[26] Z. Shen, T. Tan, G. Allison, and L. Cui, "A Customized One-Degree-of-Freedom Linkage Based Leg Exoskeleton for Continuous Passive Motion Rehabilitation," Mechanisms and Machine Science, vol. 68, pp. 518-526, 2018. https://doi.org/10.1007/978-3-030-03320-0_57

[27] D. Maneetham and P. Sutyasadi, "Controlling a Knee CPM Machine using PID and Iterative Learning Control Algorithm," TELKOMNIKA Telecommunication, Computing, Electronics and 
Control, vol. 18, no. 2, pp. $1047-1053, \quad$ April 2020. https://doi.org/10.12928/telkomnika.v18i2.14876

[28] S. Noble, G. E. P. Pearcey, C. Quartly, and E. P. Zehr, "Robot Controlled, Continuous Passive Movement of the Ankle Reduces Spinal Cord Excitability in Participants with Spasticity: A Pilot Study," Experimental Brain Research, vol. 237, pp. 3207-3220, 2019. https://doi.org/10.1007/s00221-019-05662-4

[29] R. Trochimczuk, T. Kuźmierowski, and P. Anchimiuk, "Study of CPM Device used for Rehabilitation and Effective Pain Management Following Knee Alloplasty," International Journal of Applied Mechanics and Engineering, vol. 22, no. 1, pp. 241-251, 2017. https://doi.org/10.1515/ijame-2017-0014

[30] N. Z. Azlan and N. S. Lukman, "Assist As Needed Control Strategy for Upper Limb Rehabilitation Robot in Eating Activity,” IIUM Engineering Journal, vol. 22, no. 1, pp. 298-322, January 2021. https://doi.org/10.31436/iiumej.v22i1.1480 